\title{
Rare decays and exotic states in quark flavour physics
}

\section{Justine Serrano*}

Aix Marseille Univ, CNRS/IN2P3, CPPM, Marseille, France

E-mail: serrano@cppm.in2p3.fr

Rare decays in quark flavour physics provide a unique opportunity to probe for new heavy particles beyond the Standard Model, with mass scale higher than the one directly reachable at the LHC. Recent experimental results are reviewed, with emphasize on $b \rightarrow s \ell \ell$ decays. A brief overview of the latest developments in the field of exotic states is also provided.

EPS-HEP 2017, European Physical Society conference on High Energy Physics 5-12 July 2017

Venice, Italy

${ }^{*}$ Speaker. 
Quark flavour physics is a very rich field that can be exploited both to deepen our understanding of the Standard Model (SM) and to look for new physics effects. These proceedings will focus on two different topics related to quark flavour physics: the study of rare decays and of exotic states. The main experiments contributing actively to these studies are the $e^{+} e^{-} B$-factories, Belle and Babar, that accumulated $772 \mathrm{M}$ and $467 \mathrm{M} b \bar{b}$ pairs at the $\Upsilon(4 S)$ resonance respectively, and the LHC experiments LHCb, ATLAS, and CMS. The latters benefit from the large $b \bar{b}$ production cross section in the high energy $p p$ collisions of the LHC, producing all kinds of b-hadrons, while $B$-factories can only study $B^{0}$ and $B^{ \pm}$mesons when running at the $\Upsilon(4 S)$, though in a much cleaner environment. During the Run 1 of the LHC, in 2011 (at a centre-of-mass energy of $\sqrt{s}=7 \mathrm{TeV}$ ) and 2012 (at $\sqrt{s}=8 \mathrm{TeV}$ ), LHCb has recorded $3 \mathrm{fb}^{-1}$ whereas ATLAS and CMS accumulated $25 \mathrm{fb}^{-1}$. Most of LHC results presented in this conference are based on Run 1 data with some exceptions using data from the Run 2, which started in 2015 at $\sqrt{s}=13 \mathrm{TeV}$. Section 1 presents a selection the latest experimental results concerning rare decays. Section 2 gives a brief review of recent developments regarding exotic states and spectroscopy.

\section{Rare B decays}

\subsection{Introduction}

In the SM, flavour changing neutral current (FCNC) decays, that corresponds to the transition from a down-type quark (or up-type quark) to another one of same type but different flavour, are forbidden at tree level. They can only proceed via loop diagrams, involving non-diagonal CKM matrix elements, and are therefore suppressed with respect to tree-level mediated transitions, resulting in branching fractions $(\mathscr{B})$ of the order of $10^{-5}$ or lower. These decays are sensitive probes of physics beyond the SM since the virtual SM particles circulating in the loops could be replaced by new physics particles, modifying properties of the decay such as branching fractions or angular observables. The study of FCNCs is complementary to direct new physics searches as they are sensitive to much higher scales than those directly accessible at hadron colliders.

Rare FCNC decays of b-hadrons can be described in a model-independent approach using the effective Hamiltonian

$$
\mathscr{H}_{e f f}=-\frac{4 G_{F}}{\sqrt{2}} V_{t b} V_{t q}^{*} \sum_{i}\left(C_{i} \mathscr{O}_{i}+C_{i}^{\prime} \mathscr{O}_{i}^{\prime}\right)+\text { h.c. },
$$

where $q=d, s$ for processes based on the quark level $b \rightarrow d, s$ transitions. The heavy degrees of freedom have been integrated out resulting in the short distance Wilson coefficients $C_{i}$ and the corresponding operators $\mathscr{O}_{i}$ that encode the long-distance effects. The main operators of interest are the electromagnetic operator $\mathscr{O}_{7}$ and the semileptonic operators $\mathscr{O}_{9}, \mathscr{O}_{10}$. New-physics contributions could affect the value of the Wilson coefficients $C_{7,9,10}$ or involve other operators such as chiralityflipped $\mathscr{O}_{7,9,10}^{\prime}$ or scalar and pseudoscalar $\mathscr{O}_{S, P}^{(\prime)}$. The different operators contribute differently to leptonic, semileptonic, and radiative decays. A global analysis of the experimental measurements can then result in constraints on the Wilson coefficients, that can be interpreted in the framework of specific models.

This review focuses on rare $b \rightarrow s$ transitions, excluding lepton flavour universality tests that are adressed in [1]. Rare decays of charm and strange hadrons are not covered due to lack of space but a large variety of results are available in this field from the $B$-factories, LHCb, and BES-III. 


\subsection{Leptonic decays}

The $B_{d}^{0} \rightarrow \mu^{+} \mu^{-}$and $B_{s}^{0} \rightarrow \mu^{+} \mu^{-}$channels are particularly sensitive to NP contributions in the scalar/pseudoscalar sector and have been searched for more than 25 years. The results of a combined analysis of the CMS and LHCb Run 1 data [2] revealed the first observation of the $B_{s}^{0} \rightarrow$ $\mu^{+} \mu^{-}$decays at $6.2 \sigma$. The measured branching fractions are compatible with the SM expectations, $\mathscr{B}\left(B_{s}^{0} \rightarrow \mu^{+} \mu^{-}\right)=(3.65 \pm 0.23) \times 10^{-9}$ and $\mathscr{B}\left(B_{d}^{0} \rightarrow \mu^{+} \mu^{-}\right)=(1.06 \pm 0.09) \times 10^{-10}$ [3], at 1.2 $\sigma$ level for the $B_{s}^{0}$ and $2.2 \sigma$ for the $B_{d}^{0}$ decays. The ATLAS collaboration recently presented the results of its analysis of the Run 1 data, $\mathscr{B}\left(B_{s}^{0} \rightarrow \mu^{+} \mu^{-}\right)=\left(0.9_{-0.8}^{+1.1}\right) \times 10^{-9}$ and $\mathscr{B}\left(B_{d}^{0} \rightarrow \mu^{+} \mu^{-}\right)<$ $4.2 \times 10^{-10}$ at $95 \%$ CL [4], which are in agreement with the CMS+LHCb combination and the SM predictions. These measurements are summarized in Fig. 1.

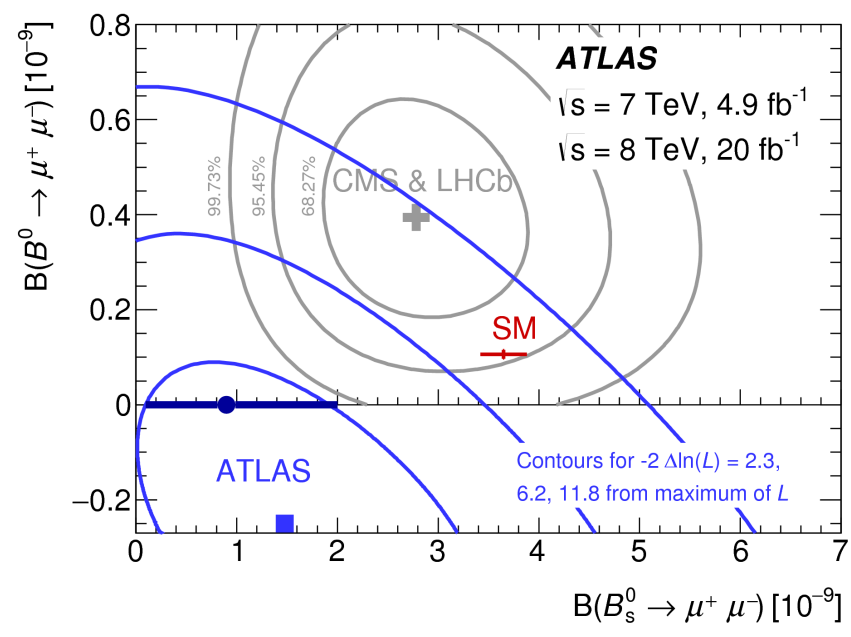

Figure 1: Likelihood contours obtained using Run 1 data by the ATLAS experiment (blue line) and the LHCb and CMS combination (black line) in the plane $\mathscr{B}\left(B_{d}^{0} \rightarrow \mu^{+} \mu^{-}\right)$vs $\mathscr{B}\left(B_{s}^{0} \rightarrow \mu^{+} \mu^{-}\right)$. The solid bullet corresponds to the maximum of the likelihood of the ATLAS dataset with the boundary of nonnegative branching fractions. The SM prediction is also shown as red cross.

Early this year, LHCb updated its analysis, by adding $1.4 \mathrm{fb}^{-1}$ of Run 2 data. The analysis has been reoptimized to reject the background from misidentified b-hadron decays and uses an improved boosted decision tree (BDT) classifier. The obtained measurements are $\mathscr{B}\left(B_{s}^{0} \rightarrow\right.$ $\left.\mu^{+} \mu^{-}\right)=\left(3.0 \pm 0.6_{-0.2}^{+0.3}\right) \times 10^{-9}$, which corresponds to the first observation of this decay by a single experiment with a significance of $7.8 \sigma$, and $\mathscr{B}\left(B_{d}^{0} \rightarrow \mu^{+} \mu^{-}\right)<3.4 \times 10^{-10}$ at 95\% CL [5]. Complementary to the branching fraction, the $B_{s}^{0} \rightarrow \mu^{+} \mu^{-}$effective lifetime also gives constraints on NP models and allows to disentangle scalar and non-scalar contributions, through $\mathscr{A}_{\Delta \Gamma}$ :

$$
\tau_{B_{s}^{0} \rightarrow \mu^{+} \mu^{-}}=\frac{\tau_{B_{s}^{0}}}{1-y_{s}^{2}} \cdot \frac{1+2 \mathscr{A}_{\Delta \Gamma} y_{s}+y_{s}^{2}}{1+\mathscr{A}_{\Delta \Gamma} y_{s}},
$$

where $\tau_{B_{s}^{0}}$ is the $B_{s}^{0}$ mean lifetime, $\tau_{B_{s}^{0}}=1.510 \pm 0.005 \mathrm{ps}$, and $y_{s}=\tau_{B_{s}^{0}} \Delta \Gamma / 2=0.062 \pm 0.0006$ $[6,7]$. Selecting candidates with a BDT output larger than 0.55 and statistically subtracting the background, the $B_{s}^{0} \rightarrow \mu^{+} \mu^{-}$distribution is fitted, as seen on Fig. 2 (right), and the value $\tau\left(B_{s}^{0} \rightarrow\right.$ $\left.\mu^{+} \mu^{-}\right)=2.04 \pm 0.44 \pm 0.05 \mathrm{ps}$ is obtained. The sensitivity to $\mathscr{A}_{\Delta \Gamma}$ is still limited but future LHC runs will be of great interest in that respect. 

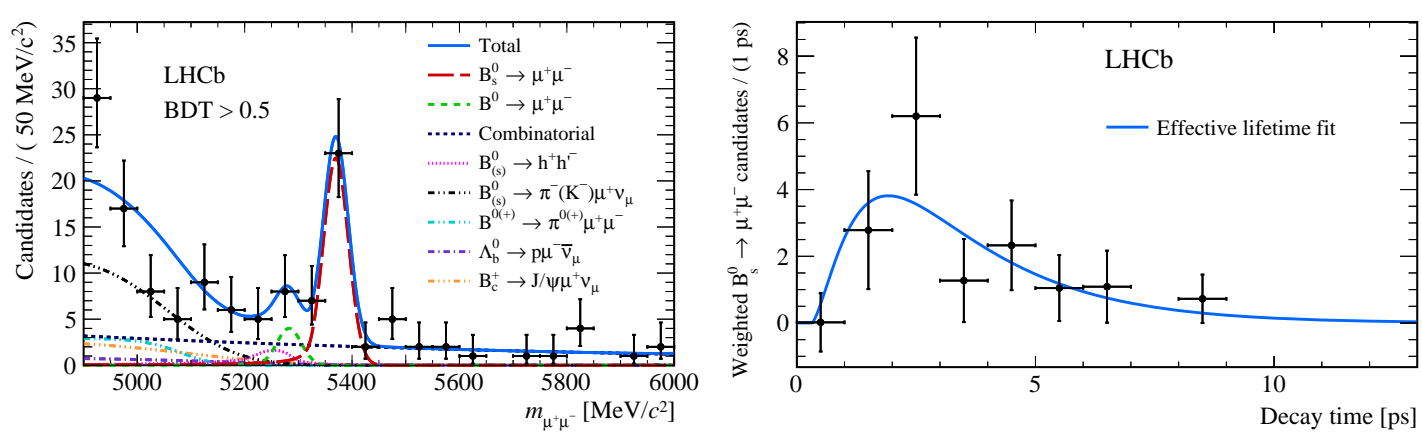

Figure 2: (left) Invariant mass distribution of the selected $B_{s}^{0} \rightarrow \mu^{+} \mu^{-}$candidates (black dots) with BDT> 0.5 . The result of the fit is overlaid, and the different components are detailed. (right) Background-subtracted $B_{s}^{0} \rightarrow \mu^{+} \mu^{-}$decay-time distribution with the fit result superimposed. Candidates with BDT $>0.55$ have been selected.

Searches for the $B$ leptonic decays into $\tau$ leptons are interesting in view of the recent hints of lepton flavour non-universality obtained by several experiments. Their branching fractions are two orders of magnitude higher than in the muon case because of the less stringent helicity suppression due to the higher lepton mass: $\mathscr{B}\left(B_{s}^{0} \rightarrow \tau^{+} \tau^{-}\right)=(7.73 \pm 0.49) \times 10^{-7}$ and $\mathscr{B}\left(B_{d}^{0} \rightarrow \tau^{+} \tau^{-}\right)=$ $(2.22 \pm 0.19) \times 10^{-8}$ [3]. However, experimental searches for these decays are complicated by the presence of at least two neutrinos in the final state due to the $\tau$ decays. Reconstructing the $\tau$ lepton in its hadronic final state $\tau^{-} \rightarrow \pi^{-} \pi^{+} \pi^{-} v_{\tau}$, the LHCb collaboration has obtained the first limit on the $B_{s}^{0} \rightarrow \tau^{+} \tau^{-}$branching ratio at $6.8 \times 10^{-3}$ (95\% CL) and the best limit on $\mathscr{B}\left(B_{d}^{0} \rightarrow \tau^{+} \tau^{-}\right)$, at $2.1 \times 10^{-3}(95 \% \mathrm{CL})$ [8], using Run 1 data. These limits are orders of magnitudes higher than the SM predictions but open the perspective of other rare decays into $\tau$ leptons being studied at LHC experiments.

\subsection{Semileptonic decays}

Semileptonic $b \rightarrow$ sl $\ell$ decays give access to a wide range of observables, commonly measured in bins of the dilepton invariant mass square, $q^{2}$. Their theoretical predictions are affected by hadronic uncertainties arising from the form factors that are computed using lattice QCD or light cone sum-rule techniques, depending on the $q^{2}$ region, and subleading $\Lambda_{Q C D} / m_{b}$ corrections to QCDF. From an experimental point of view, the muonic modes are the easiest to reconstruct for the LHC experiments ${ }^{1}$, while the $B$-factories are using both electrons and muons. Decays into $\tau$ are much more challenging and only the Babar experiment has obtained a limit on $\mathscr{B}\left(B^{+} \rightarrow K^{+} \tau^{+} \tau^{-}\right)$ at $2.25 \times 10^{-3}(90 \% \mathrm{CL})[10]$.

Regarding the $b \rightarrow s \mu^{+} \mu^{-}$modes, an interesting picture emerges from the differential branching fraction measurements by the LHCb collaboration, where the experimental values tend to be lower than the SM predictions, especially below the charmonium resonances [11, 12, 13, 14]. As illustrated on Fig. 3, this effect is present both in mesonic and baryonic sectors and the largest discrepancy is seen in the $B_{s} \rightarrow \phi \mu^{+} \mu^{-}$channel, where the deviation is at the level of $3 \sigma$. This trend has also been seen by the CMS collaboraton in the $B^{0} \rightarrow K^{* 0} \mu^{+} \mu^{-}$decay [15].

\footnotetext{
${ }^{1} \mathrm{LHCb}$ has also performed an angular analysis of the $B^{0} \rightarrow K^{* 0} e^{+} e^{-}$decay at low $q^{2}$ [9].
} 

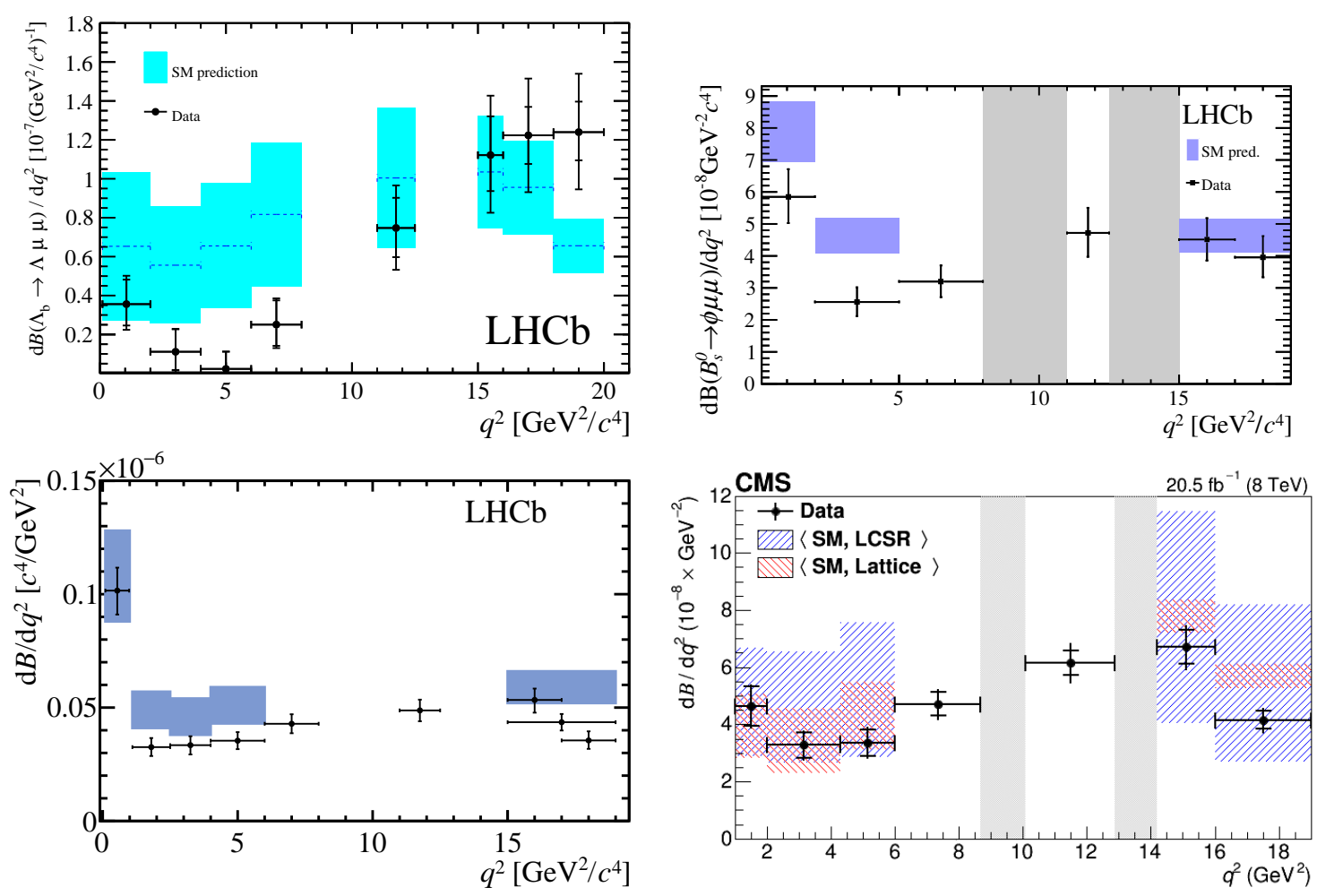

Figure 3: Differential branching fraction as function of $q^{2}$ measured by LHCb for (top left) $\Lambda_{b} \rightarrow$ $\Lambda \mu^{+} \mu^{-}$[12], (top right) $B_{S} \rightarrow \phi \mu^{+} \mu^{-}$[13], (bottom left) $B^{0} \rightarrow K^{* 0} \mu^{+} \mu^{-}$[14]; and by CMS for (bottom right) $B^{0} \rightarrow K^{* 0} \mu^{+} \mu^{-}[15]$.

The $B \rightarrow K^{*} \ell^{+} \ell^{-}$decays, where $\ell=e, \mu$, are the most extensively studied modes [15, 16, 17, $18,19,20]$. The corresponding differential decay rate can be written as

$$
\frac{1}{d \Gamma / d q^{2}} \cdot \frac{d^{4} \Gamma\left[\bar{B}^{0}+B^{0}\right]}{d \cos \theta_{\ell} d \cos \theta_{K} d \phi d q^{2}}=\frac{9}{32 \pi} \sum_{i} S_{i}\left(q^{2}\right) f_{i}\left(\cos \theta_{\ell}, \cos \theta_{K}, \phi\right)
$$

where the angular observables $S_{i}$ are functions of the Wilson coefficients. Other observables with reduced form-factors uncertainties can be formed from ratios of $S_{i}$, in particular $P_{5}^{\prime}=\frac{S_{5}}{\sqrt{F_{L}\left(1-F_{L}\right)}}$, where $F_{L}$ is the fraction of longitudinally polarized $K^{*}$. Depending on available signal yields, the experiments use different analysis techniques to access these angular observables. All measurements are in good agreement with the SM predictions, except in the case of the $P_{5}^{\prime}$ observable, for which LHCb, Belle and ATLAS find a deviation in the region $4<q^{2}<8 \mathrm{GeV}^{2} / c^{4}$ at the level of $2-3 \sigma$. A summary of the experimental results is shown on Fig. 4. The Belle collaboration has also split this measurement for electrons and muons and find that the discrepancy is at the $2.6 \sigma$ level for the muons while being at $1.1 \sigma$ for the electrons, pointing, here again, to an anomaly in the muon sector. These discrepancies can be interpreted as new physics contribution to the $C_{9}$ Wilson coefficient (see [23]).

\subsection{Radiative decays}

At leading order in the SM, the $b \rightarrow d \gamma$ and $b \rightarrow s \gamma$ transitions are mediated through the 


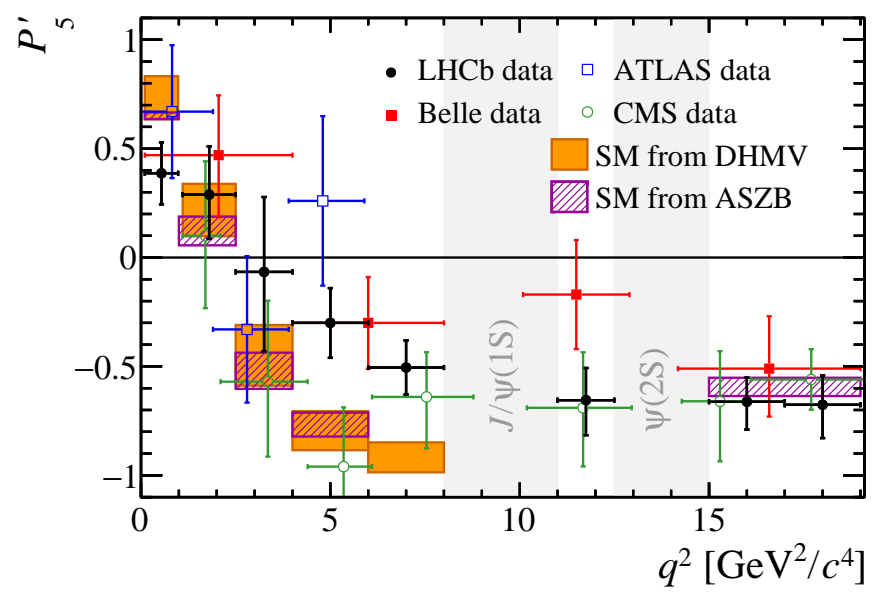

Figure 4: The $P_{5}^{\prime}$ observable as function of $q^{2}$ obtained by LHCb, Belle, ATLAS and CMS in $B \rightarrow K^{*} \ell^{+} \ell^{-}$ decays (points with error bars). LHC experiments use only $B^{0} \rightarrow K^{* 0} \mu^{+} \mu^{-}$whereas Belle combines electron and muon final states and both charged and neutral modes. The coloured boxes shows the SM predictions based on form factors calculations from Lattice QCD (Ref. [21]) and Light cone sum rules (Ref. [22]).

electromagnetic dipole operator $\mathscr{O}_{7}$. The chirality-flipped operator $\mathscr{O}_{7}^{\prime}$ is suppressed by $m_{s, d} / m_{b}$. Sensitivity to new physics appears through measurements of branching fractions, photon helicity, or $C P$ and isospin asymmetries.

At this conference, the Belle collaboration reported a new analysis of the decay $B \rightarrow K^{*} \gamma$, founding the first evidence at $3.1 \sigma$ of isospin asymmetry, defined as

$$
\Delta_{0+}=\frac{\Gamma\left(B^{0} \rightarrow K^{* 0} \gamma\right)-\Gamma\left(B^{+} \rightarrow K^{*+} \gamma\right)}{\Gamma\left(B^{0} \rightarrow K^{* 0} \gamma\right)+\Gamma\left(B^{+} \rightarrow K^{*+} \gamma\right)} .
$$

The measurement yields $\Delta_{0+}=(+6.2 \pm 1.5 \pm 0.6 \pm 1.2) \%$, where the uncertainties are statistical, systematic and from the fraction of $B^{+} B^{-}$to $B^{0} \bar{B}^{0}$ production in $\Upsilon(4 S)$ decays [24]. This result is in agreement with the SM predictions, which range from $2 \%$ to $8 \%$ with a typical uncertainty of $2 \%$. The Belle collaboration also obtained the most precise $C P$ asymmetry $\left(A_{C P}\right)$ measurements for the charged and neutral modes, and the difference between the two, $\Delta A_{C P}=A_{C P}\left(B^{+} \rightarrow\right.$ $\left.K^{*+} \gamma\right)-A_{C P}\left(B^{0} \rightarrow K^{* 0} \gamma\right)=(+2.4 \pm 2.8 \pm 0.5) \%$, which is consistent with zero. These results, still statistically limited, will improve with the upcoming Belle II experiment. 


\section{Exotic and new states}

Since the first exotic states discovered by the Belle collaboration in 2003, about 30 new exotic hadrons, mainly charmoniumlike or bottomoniumlike, have been seen by different experiments. Their theoretical interpretation is still unclear. Several models, such as molecular or hybrid, are considered, but they are unsucessful at describing all the observed states.

One of the most important experimental result of the last years is the discovery of two states of minimal quark content $c \bar{c} u u d$ seen in an amplitude analysis of $\Lambda_{b} \rightarrow J / \Psi p K$ decays by LHCb [25]. The heavier of these states, the $P_{c}(4450)$ has a width of $\sim 40 \mathrm{MeV} / c^{2}$ and its Argand diagram is consistent with a resonance. The lighter one is broader $\left(\sim 200 \mathrm{MeV} / c^{2}\right)$, and its Argand diagram is less conclusive. New decay modes are being studied in order to confirm the pentaquark nature of these states such as $\Lambda_{b} \rightarrow J / \Psi p \pi$ [26] and $\Lambda_{b} \rightarrow \chi_{c} p K$ [27]. An angular analysis of the $\Lambda_{b} \rightarrow$ $J / \Psi p \pi$ mode using Run 1 data by the LHCb collaboration shows that exotic states are needed to described the data but the statistics is still too limited to identify them. Amplitude analyses of other decay modes will be performed in the future using larger data samples.

Regarding tetraquark states, a recent analysis of the $B \rightarrow J / \Psi \phi K$ decay by LHCb established the existence of four states : the X(4140), already reported by CDF, D0 and CMS, the X(4274), previously seen by CDF and CMS, and two new states, the X(4500) and the X(4700) [28]. These four resonances are observed with significances larger than $5 \sigma$ and can be clearly seen on Fig. 5 [28].

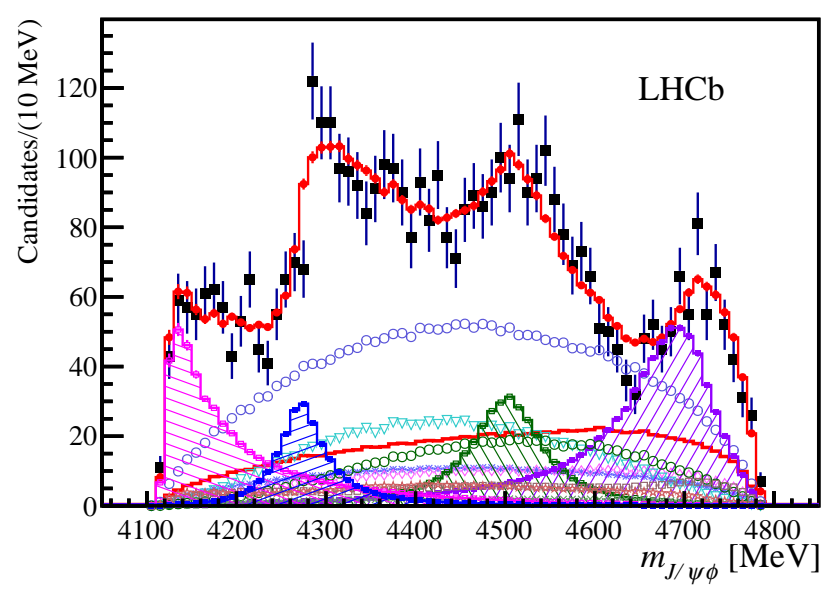

Figure 5: The $J / \Psi \phi$ invariant masses of the $B^{+} \rightarrow J / \Psi \phi K^{+}$candidates (black points) compared with the results of the default amplitude fit (red points with error bars). The tetraquarks contributions X(4140), $\mathrm{X}(4274), \mathrm{X}(4500)$ and X(4700) are shown by pink, blue, green, and purple histograms, respectively. See Ref. [28] for the definition of other individual fit components.

The last result presented here concerns hadron spectroscopy. The LHCb collaboration has presented at this conference the observation of a new baryon containing two charm quarks, the $\Xi_{c c}^{++}$baryon. This is not a so-called exotic state as the quark model predicts the existence of three double-charm baryons: the $\Xi_{c c}^{++}$with a quark content $c c u$, the $\Xi_{c c}^{+}$with quark content $c c d$ and the $\Omega_{c c}^{+}$with quark content $c c s$. Being isosin partners, the $\Xi_{c c}^{++}$and $\Xi_{c c}^{+}$are expected to have masses differing by only a few $\mathrm{MeV} / c^{2}$, but the lifetime of the $\Xi_{c c}^{++}$should be about three times larger 
than the one of the $\Xi_{c c}^{+}$. The observation of the $\Xi_{c c}^{+}$baryon was reported previously by the SELEX experiment $[29,30]$, but not confirmed by FOCUS, Babar or Belle. The LHCb collaboration used $1.7 \mathrm{fb}^{-1}$ of data taken in 2016 to look for the decay of a $\Xi_{c c}^{++}$into $\Lambda_{c}^{+} K^{-} \pi^{+} \pi^{+}$, with $\Lambda_{c}^{+} \rightarrow$ $p K^{-} \pi^{+}$and found a signal yield of $313 \pm 33$ events, as seen on Fig. 6 [31]. The measured mass of $3621.40 \pm 0.72 \pm 0.27 \pm 0.14 \mathrm{MeV} / c^{2}$, where the uncertainties are statistical, systematic, and from the known $\Lambda_{c}^{+}$mass, is $\sim 100 \mathrm{MeV} / c^{2}$ larger than the one reported by SELEX for the $\Xi_{c c}^{+}$, disfavouring the interpretation of the SELEX structure as the $\Xi_{c c}^{+}$state. This constitutes the first observation of a baryon containing two heavy quarks and provides a very interesting tool to probe QCD.

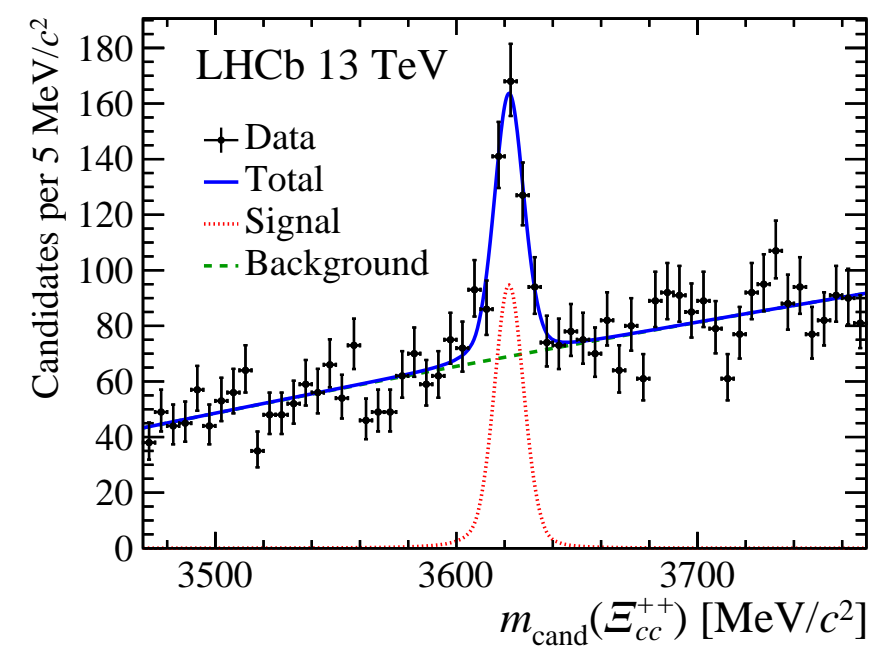

Figure 6: Invariant mass distribution of $\Lambda_{c}^{+} K^{-} \pi^{+} \pi^{+}$candidates with fit projections overlaid.

\section{Conclusion}

Recent results in quark flavour physics have been presented. Concerning rare decays, though most of experimental measurements are in agreement with SM predictions, intringuing tensions exist in $b \rightarrow s \mu^{+} \mu^{-}$decays, both in differential branching fractions and angular observables. These deviations become even more exciting when put in perspective with the hints of lepton flavour universality violation seen in $R\left(K^{(*)}\right)$ and $R\left(D^{(*)}\right)$. On the exotic side, more new states are being discovered, adding information to the puzzling picture of this new class of hadrons.

The coming years are expected to be of great interest on the experimental side. The LHC experiments are just in the middle of the Run 2 data-taking, benefiting from a larger $b \bar{b}$ cross-section thanks to the energy increase. Belle II will start taking data in 2018, with a final target of $50 \mathrm{ab}^{-1}$. The NA62 experiment at CERN, studying rare $K^{+}$decays, is also taking data and the first physics results are expected in the coming months. At JPARC, an upgrade of the KOTO experiment, which is looking for $K^{0} \rightarrow \pi^{0} v \bar{v}$ decays, is ongoing and the SM sensitivity is expected to be reachable by 2021. Searches for lepton flavour violating muon decays will also be significantly improved with the future experiments MEGII, COMET, and Mu2e. This broad flavour physics program will therefore certainly allow to drastically constrain new physics phase space or discover it. 


\section{References}

[1] U. Egede, "Highlights from the LHCb experiment", in these proceedings.

[2] V. Khachatryan et al. [CMS and LHCb Collaborations], Nature 522, 68 (2015) doi:10.1038/nature14474 [arXiv:1411.4413 [hep-ex]].

[3] C. Bobeth, M. Gorbahn, T. Hermann, M. Misiak, E. Stamou and M. Steinhauser, Phys. Rev. Lett. 112 (2014) 101801 doi:10.1103/PhysRevLett.112.101801 [arXiv:1311.0903 [hep-ph]].

[4] M. Aaboud et al. [ATLAS Collaboration], Eur. Phys. J. C 76 (2016) no.9, 513 doi:10.1140/epjc/s10052-016-4338-8 [arXiv:1604.04263 [hep-ex]].

[5] R. Aaij et al. [LHCb Collaboration], Phys. Rev. Lett. 118, no. 19, 191801 (2017) doi:10.1103/PhysRevLett.118.191801 [arXiv:1703.05747 [hep-ex]].

[6] Y. Amhis et al., arXiv:1612.07233 [hep-ex].

[7] C. Patrignani et al. [Particle Data Group], Chin. Phys. C 40, no. 10, 100001 (2016). doi:10.1088/1674-1137/40/10/100001

[8] R. Aaij et al. [LHCb Collaboration], Phys. Rev. Lett. 118, no. 25, 251802 (2017) doi:10.1103/PhysRevLett.118.251802 [arXiv:1703.02508 [hep-ex]].

[9] R. Aaij et al. [LHCb Collaboration], JHEP 1504, 064 (2015) doi:10.1007/JHEP04(2015)064 [arXiv:1501.03038 [hep-ex]].

[10] J. P. Lees et al. [BaBar Collaboration], Phys. Rev. Lett. 118, no. 3, 031802 (2017) doi:10.1103/PhysRevLett.118.031802 [arXiv:1605.09637 [hep-ex]].

[11] R. Aaij et al. [LHCb Collaboration], JHEP 1406 (2014) 133 doi:10.1007/JHEP06(2014)133 [arXiv:1403.8044 [hep-ex]].

[12] R. Aaij et al. [LHCb Collaboration], JHEP 1506 (2015) 115 doi:10.1007/JHEP06(2015)115 [arXiv:1503.07138 [hep-ex]].

[13] R. Aaij et al. [LHCb Collaboration], JHEP 1509 (2015) 179 doi:10.1007/JHEP09(2015)179 [arXiv:1506.08777 [hep-ex]].

[14] R. Aaij et al. [LHCb Collaboration], JHEP 1611 (2016) 047 doi:10.1007/JHEP11(2016)047 [arXiv:1606.04731 [hep-ex]].

[15] V. Khachatryan et al. [CMS Collaboration], Phys. Lett. B 753, 424 (2016) doi:10.1016/j.physletb.2015.12.020 [arXiv:1507.08126 [hep-ex]].

[16] R. Aaij et al. [LHCb Collaboration], JHEP 1602, 104 (2016) doi:10.1007/JHEP02(2016)104 [arXiv:1512.04442 [hep-ex]].

[17] The ATLAS collaboration [ATLAS Collaboration], ATLAS-CONF-2017-023.

[18] CMS Collaboration [CMS Collaboration], CMS-PAS-BPH-15-008.

[19] S. Wehle et al. [Belle Collaboration], Phys. Rev. Lett. 118, no. 11, 111801 (2017) doi:10.1103/PhysRevLett.118.111801 [arXiv:1612.05014 [hep-ex]].

[20] J. P. Lees et al. [BaBar Collaboration], Phys. Rev. D 93, no. 5, 052015 (2016) doi:10.1103/PhysRevD.93.052015 [arXiv:1508.07960 [hep-ex]].

[21] R. R. Horgan, Z. Liu, S. Meinel and M. Wingate, PoS LATTICE 2014, 372 (2015) [arXiv:1501.00367 [hep-lat]]. 
[22] A. Bharucha, D. M. Straub and R. Zwicky, JHEP 1608, 098 (2016) doi:10.1007/JHEP08(2016)098 [arXiv:1503.05534 [hep-ph]].

[23] S. Gori, "Flavour physics theory", in these proceedings.

[24] T. Horiguchi et al. [Belle Collaboration], arXiv:1707.00394 [hep-ex].

[25] R. Aaij et al. [LHCb Collaboration], Phys. Rev. Lett. 115, 072001 (2015) doi:10.1103/PhysRevLett.115.072001 [arXiv:1507.03414 [hep-ex]].

[26] R. Aaij et al. [LHCb Collaboration], Phys. Rev. Lett. 117, no. 8, 082003 (2016) Addendum: [Phys. Rev. Lett. 117, no. 10, 109902 (2016)] Addendum: [Phys. Rev. Lett. 118, 119901 (2017)] doi:10.1103/PhysRevLett.118.119901, 10.1103/PhysRevLett.117.082003, 10.1103/PhysRevLett.117.109902 [arXiv:1606.06999 [hep-ex]].

[27] R. Aaij et al. [LHCb Collaboration], Phys. Rev. Lett. 119, no. 6, 062001 (2017) doi:10.1103/PhysRevLett.119.062001 [arXiv:1704.07900 [hep-ex]].

[28] R. Aaij et al. [LHCb Collaboration], Phys. Rev. Lett. 118, no. 2, 022003 (2017) doi:10.1103/PhysRevLett.118.022003 [arXiv:1606.07895 [hep-ex]].

[29] M. Mattson et al. [SELEX Collaboration], Phys. Rev. Lett. 89, 112001 (2002) doi:10.1103/PhysRevLett.89.112001 [hep-ex/0208014].

[30] A. Ocherashvili et al. [SELEX Collaboration], Phys. Lett. B 628, 18 (2005) doi:10.1016/j.physletb.2005.09.043 [hep-ex/0406033].

[31] R. Aaij et al. [LHCb Collaboration], arXiv:1707.01621 [hep-ex]. 